Cinémas

Revue d'études cinématographiques

Journal of Film Studies

\title{
Travestissement et paternité : la masculinité remade in the USA
}

\section{Anne-Marie Picard}

Volume 1, numéro 1-2, automne 1990

URI : https://id.erudit.org/iderudit/1000994ar

DOI : https://doi.org/10.7202/1000994ar

Aller au sommaire du numéro

Éditeur(s)

Cinémas

ISSN

1181-6945 (imprimé)

1705-6500 (numérique)

Découvrir la revue

Citer cet article

Picard, A.-M. (1990). Travestissement et paternité : la masculinité remade in the USA. Cinémas, 1(1-2), 114-131. https://doi.org/10.7202/1000994ar
Résumé de l'article

Les films Trois hommes et un couffin de Coline Serreau et Three Men and a Baby de Leonard Nimoy sont analysés ici de façon comparative. L'auteure explique la façon dont la version américaine fait une « re-vision » du regard attendri que Serreau portait sur les nouveaux hommes nés des mouvements et des analyses féministes. La nouvelle masculinté (non phallique) illustrée dans le film français est totalement abolie et remplacée par l'idéologie patriarcale américaine. 


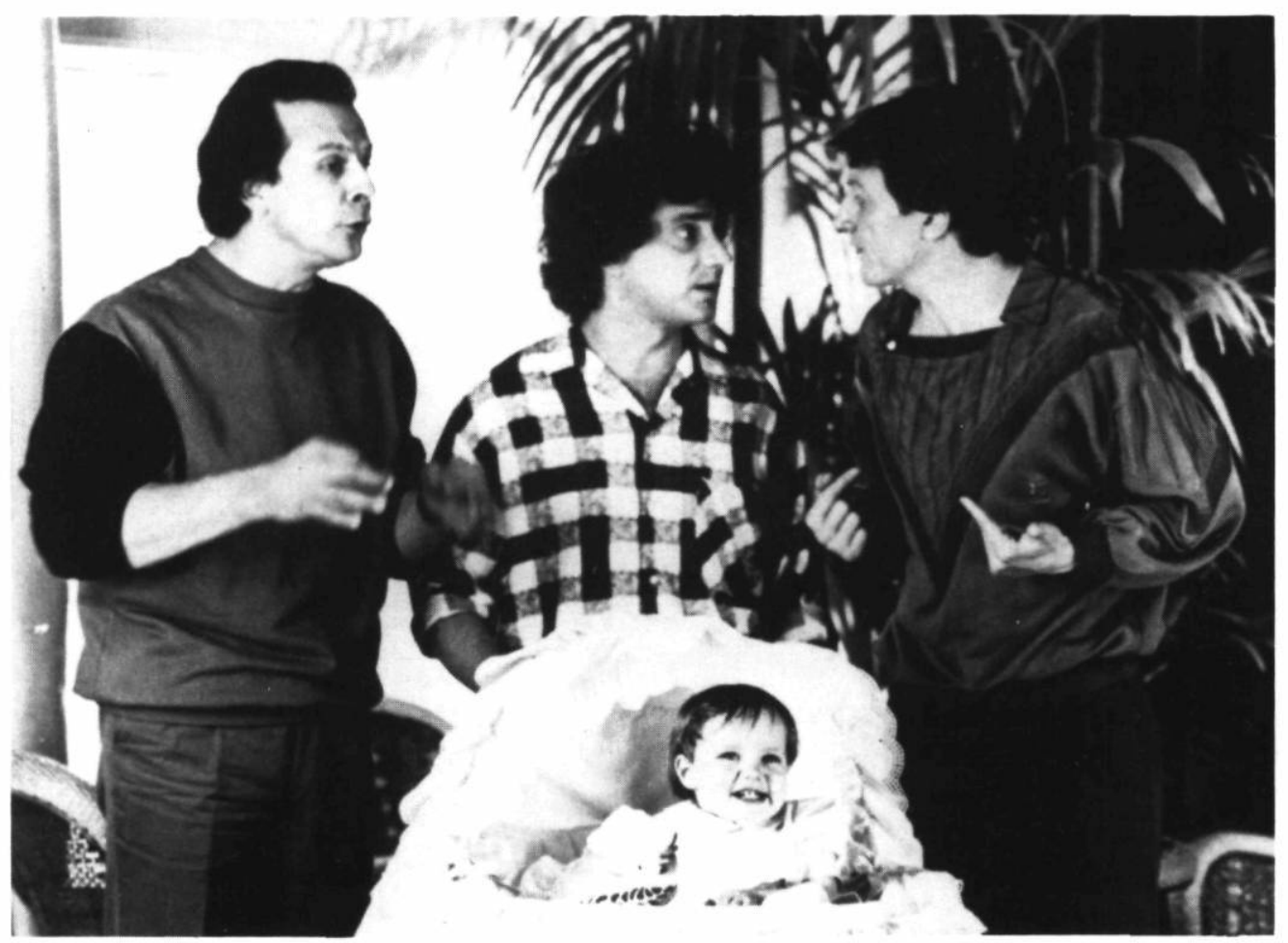

Trois hommes et un couffin de Coline Serreau (1984)

Coll. Cinémathèque québécoise 


\title{
Travestissement et paternité: la masculinite remade in the USA
}

\section{Anne-Marie Picard}

\begin{abstract}
RÉSUMÉ
Les films Trois hommes et un couffin de Coline Serreau et Three Men and a Baby de Leonard Nimoy sont analysés ici de façon comparative. L'auteure explique la façon dont la version américaine fait une «re-vision» du regard attendri que Serreau portait sur les nouveaux hommes nés des mouvements et des analyses féministes. La nouvelle masculinté (non phallique) illustrée dans le film français est totalement abolie et remplacée par l'idéologie patriarcale américaine.
\end{abstract}

\begin{abstract}
Coline Serreau's film Trois hommes et un couffin is analysed in comparison to Leonard Nimoy's Three Men and a Baby. The author shows how the American version is a "re-vision" of the tender gaze cast by Serreau on the "new men", men born in the wake of the feminist movement and its analyses. The new, non-phallic masculinity illustrated in the French film is totally eliminated and replaced by American patriarchal ideology.
\end{abstract}

Des lecteurs de Foucault à ceux de Kristeva, de la déconstruction à la «nouvelle cuisine», la francité resplendit, ces temps-ci, des lectures américaines enthousiastes. Le remake hollywoodien participe allégrement de cette réécriture de la pensée des autres. Le phénomène a quelque chose d'agaçant et de fascinant à la fois pour qui a la tête façonnée en France: agaçant parce que les remakes sont tellement trempés de la sauce USA qu'ils glissent entre les doigts, qu'ils échappent - ils sont toujours autre chose; et fascinant parce 
qu'on se dit qu'Hollywood, ce monstre de l'américanisation tentaculaire, n'a peut-être plus d'herbe autochtone à se mettre sous la dent et que cela annonce ou sa belle mort d'animal préhistorique, ou la capacité gigantesque d'adaptation de son système digestif.

Trois hommes et un couffin, de Coline Serreau, a battu Rambo au box-office français en 1984. Du jamais-vu! Ça pleurait au maximum dans les salles. Les papas et les mamans de France, appuyés dans leur lutte contre la dénatalité par des campagnes de publicité mirobolantes, riaient aux éclats du mâle français en perte de virilité, mais en gain de tendresse. «Le nouveau papa est arrivé!» Quelque chose de l'inconscient collectif trouvait alors satisfaction et parole, dans les clowneries, les hésitations, les déficiences de trois bourgeois parisiens face à ce bébé rose gigotant.

Le projet d'un remake américain voit le jour. Coline Serreau refuse d'y participer ${ }^{1}$. Leonard Nimoy et son équipe se mettent alors à l'œuvre pour tirer profit de cette tendance nouvelle: celle du mâle qui assume. Kramer vs Kramer, Mr Mom... la liste s'allonge, la leçon est reçue: «Faites des bébés!» M. Spock, aux aguets (question de rentabiliser l'idée), a le stylo pesant: il nous rature des grands bouts de scénario, en réécrit d'autres, nous réaménage tout cela pour satisfaire les horizons d'attente du cow-boy des grands déserts de l'imaginaire hollywoodien: il y faut du muscle, de la consistance. Ainsi, dans le remake, les additions, les glissements semblent être là à la fois pour censurer et pour combler une angoisse qui va, par là-même, transparaître. Qu'en est-il, pour le sujet masculin américain, de la recherche du père? Où se déplace la masculinité? Où rejoue-t-elle le statu quo de ses fantasmes? La réponse américaine à la question posée par une femme française prend les allures d'un aveu.

\section{Le père freudien est question ${ }^{2}$}

La question du père, chez Freud, ne se pose pas en termes d'une psychologie du père mais comme la question du lien au père: c'est ce lien (avant d'être rapport, subjectif donc) qui fait problème.

Il s'agit évidemment du fait qu'on sait toujours plus sûrement qui est notre mère que notre père. Cette «clause de l'incertitude potentielle du père est méditée par l'inconscient du rejeton» (Assoun, p. 39) et l'amènera à penser, à faire des hypothèses. Ainsi ce lien en forme de question est, phylogénétiquement, la condition de toute pensée déductive, et pour Freud, le fait que l'on porte le nom du père prouve l'importance culturelle de celui-ci:

«(...) la maternité [dit Freud] est attestée par le témoignage des sens, tandis que la paternité est une hypothèse (...) édifiée sur une conclusion et sur une présupposition.» Il faut comprendre [commente Paul- 
Laurent Assoun] que l'évidence sensible (sensuelle) de la mère contraste avec la dynamique intellectuelle sur laquelle la paternité embraye. Du fait même que l'évidence du père ne crève pas les yeux (...) s'ouvre un espace de jeu. (...) L'essentiel [pour Freud] est donc que le père donne à penser. Ce qui atteste qu'il est tout sauf une nature ou une «donnée». Bref, il y a un lien secret et déterminant entre l'incertitude ontologique de la paternité et sa fécondité dans l'ordre de la pensée (p. 38-39).

Et ainsi, c'est parce que le père est problématique dans son être, qu'il va être, par la romantisation familiale de sa progéniture, remplacé, évacué et «relevé» en même temps comme Idéal du moi, père aimant et protecteur, divinité: le père meurt comme corps pour advenir comme Dieu. En d'autres termes, la stratégie consiste à «sauver le père au nom du père quitte à en immoler le corps pour en exalter l'esprit» (Assoun, p. 39).

Quand on joue à Candide ceci est déjà problématique. Car dans les techniques d'accouchement actuels où la mère est parfois endormie, le risque d'échange de bébés est possible réellement et comme question inconsciente potentielle du sujet. La mère peut aussi être incertaine. Mais sans doute ne s'agit-il pas de ce genre de savoir-là, de savoir objectif, biologique, mais de savoir de corps (Freud parle de «témoignage des sens»). Il s'agirait donc de «sensations de certitude» créées par les soins maternels, par le lien établi par la demande et sa satisfaction: la mère est alors non plus la génitrice mais la personne qui remplit la fonction nourricière. Il y a confusion, chez Freud, et ceux qui parlent de cette incertitude du seul géniteur mâle, entre mère porteuse et mère fonctionnelle: entre la provenance topographique, si l'on peut dire, et l'investissement amoureux, entre filiation biologique et affiliation sociale ${ }^{3}$.

Qui profite de cet adage de la certitude de la maternité comme attachée à une fonction sociobiologique immuable, à un lieu immanent, corps, caverne, demeure? Quelle angoisse est ainsi résolue? Sans cette certitude-là, celle de la place de la mère comme ancrage, objet du désir, point stable, le petit sujet de la métapsychologie freudienne ne sait plus d'où partir pour sa quête d'une identité sexuée et d'un désir qui lui soient propres. Il nous est dit, en effet, que le petit Edipe, dans sa romantisation familiale, conserve la mère comme réelle, avant de rétrograder son père réel et de s'en inventer un autre, qui n'est plus corps (le corps du père est par là même déchu) mais point de vue. La mère n'est donc jamais «rétrogradée» de façon durable, clivée en mère réelle et en mère idéale, «tant elle s'égale à sa propre nature» (Assoun, p. 39). Une mère est une mère. Il n'y a donc, pour le sujet œdipien freudien, 
aucune chance d'idéaliser la mère, de s'en construire une imago, une figure qui prendrait la place de la mère réelle, car elle est là d'où l'on vient.

Et si la génitrice est «connaissable» parce que l'enfant sort de son corps, qui donc connaît? L'enfant? Ou est-ce le mari? La loi sociale et matrimoniale? La mère freudienne apparaît donc comme corps maternant seulement (la gestation et la parturition étant présupposées à ce maternage): la mère est essence, donnée, elle n'est pas fonction ou position. Freud et les autres oublient que c'est la mère qui sait, qui est la seule détentrice du savoir. Elle est aussi celle qui nomme le père, qui fait de son mari le père de ses enfants en prenant son nom ou, implicitement, en lui laissant assumer la paternité civique, en le laissant reconnaître l'enfant ${ }^{4}$.

C'est donc l'angoisse masculine quant à sa propre généalogie et à sa problématique d'identification à un père incertain qui semble faire parler la psychanalyse freudienne. Le père comme question est la réponse fondatrice de la psychanalyse et son angoisse. Est-ce parce qu'elle nous arrive à travers les cadres fantasmatiques de sujets masculins, qui sont à la fois juges et partis? Où déceler les traces de leur vœu pieux?

Distinguons, très succinctement ici, les trois niveaux (ou plutôt les trois cadres) d'analyse qui permettront de différencier les enjeux des positions imaginaires dans le «spectatoriat»:

-1 er niveau: nos trois hommes sont les représentations de trois sujets masculins en quête d'une nouvelle identité, d'une nouvelle paternité responsable mais jouissive. Nous sommes là dans le leurre du cinéma comme piège à réalité, dans l'illusion de l'image comme miroir.

$-2^{\mathrm{e}}$ niveau: les trois hommes sont des personnages, donc des incarnations dans des corps d'acteurs des fantasmes de l'auteur(e). Ce qui se trouve représenté à ce niveau d'analyse, c'est l'espace vide du sujet désirant (qui ne correspond pas forcément au lieu du regard de la caméra), lui aussi en quête de son désir propre et qui, dans son roman familial, compose des figures humaines, des imagos, comme autant de succédanés paternels et/ou maternels. Le film est pris à ce niveau comme une projection fantasmatique individuelle et/ou collective: elle peut en effet saisir le pouls d'un inconscient collectif à un moment donné et prendre une dimension mythique comme mise en cohérence d'une question non encore formulée (ce qui s'est passé avec Trois hommes et un couffin).

- 3e niveau: le spectateur et l'auteur occupant a posteriori le même espace dans la scène du fantasme, l'identification va jouer avec ce lieu d'où on me montre l'autre. Mais l'autre est aussi moi, reconnu. Se produit un possible va-et-vient entre les deux positions des niveaux 1 et 2 . Je suis l'autre du miroir, et me retournant, je 
vois l'autre (tiers, place de l'auteur) qui regarde avec moi ce que je regarde: triangulation du jeu narcissique mis en lumière par le stade du miroir lacanien ${ }^{5}$.

Comment ne pas se laisser leurrer par le miroitement? Comment analyser un film sans que les fantasmes de chacun ne s'inscrivent dans le réalisme trompeur de l'icône? Un signe est le signe d'une absence et on peut encore se poser la question: qu'est-ce qu'un signe au cinéma ${ }^{6}$ ? Un signifiant imaginaire, dit Christian Metz. Un signe de l'imaginaire? De même, la théorisation de ces enjeux, permise par la "vérité» psychanalytique, se trouve, elle aussi, être le cadre des scénarios familiaux et angoissés de ses maîtres. Comment alors se servir de la psychanalyse? Ou plutôt, comment se faire servir par la psychanalyse?

\section{Paterner, c'est faire la femme: l'expérimentation}

Dans Three Men and a Baby (1987), le décor est planté dès le générique: l'artiste du groupe des trois finit la fresque murale de l'entrée, une fresque à la gloire des habitants du lieu. On assiste alors, en accéléré, à la circulation des femmes dans le temple que l'architecte, le dessinateur et l'acteur se sont érigés, ont érigé à la masculinité rayonnante de la classe professionnelle de New York. Trois beaux jeunes hommes à l'allure puérile, dont deux insatiables sexuellement, Peter et Jack, et un autre, le sensible Michael, qui joue plutôt au grand frère avec les femmes. C'est ce dernier qui incarne le sentiment, les deux autres pouvant se laisser aller à la virilité pure: les durs ont besoin d'un sensible pour exister.

Ainsi dans la scène d'ouverture, une fête d'anniversaire pour Peter, ils sont héroïsés comme les mâles américains de la cité. Alors que le film français ne fait que suggérer l'ambiance garçonnière de l'appartement, la version américaine n'en finit plus de s'attarder à la virilité de ces hommes, mise sur un piédestal par «les plus belles filles de New York», les serviteurs aux gants blancs, et le décor yuppie. Par exemple, en fin de soirée, Jack, comme le Jacques du film français, a une nouvelle conquête dans sa chambrée; le couple revient ensuite à la cuisine ${ }^{7}$. Jack plaisante alors sur sa future folle nuit et dit: "Tough job but it's got to be done." Mimant la bravoure du cow-boy, il rejoint ses pénates; les deux colocataires commentent: "Amazing! The guy is a giant gland!" C'est la reconnaissance mutuelle du désir continu et infatigable du mâle.

Pourquoi trois hommes? Parce que, pour l'expérience qui va avoir lieu, un seul homme aurait fait «cas particulier», et deux hommes, couple homosexuel. Les trois hommes vont tout à coup être obligés de faire des gestes de femmes ${ }^{8}$. Le risque est grand pour les sujets en question. L'agressivité montrera le niveau de stress encouru par les sujets de l'expérience. Car, au-delà du rôle 
du père nourricier, c'est toute la question de l'identité masculine qui est mise en scène. Une identité qui va se démanteler et se reconstituer sous l'œil implacable de l'autre homme, le rival, le surmoi viril possesseur du phallus. Le colocataire incarne ici la provenance, toujours imaginaire, du regard phallique. C'est cela qui est en jeu; et faire la femme sous ce regard-là, représentant l'identité masculine, est menaçant: car le ridicule tue le mâle.

L'homme est d'abord fils de femme dans nos sociétés patriarcales mais matrilinéaires. En lui, il y a deux mouvements contradictoires: la nostalgie de la féminité maternelle et la peur de sa propre féminité. Toucher le corps d'un enfant, en prendre soin, c'est entrer dans le modèle féminin de maternage, c'est mimer sa propre mère. Repenser la paternité comme un modèle symétrique à la maternité, c'est donc, comme le dit Delaisi de Parseval ${ }^{9}$, provoquer une crise de la masculinité: «D'abord, parce que cela remet en cause la valeur de la maternité; mais aussi parce que cela révèle la bisexualité de l'être humain» et provoque «une interrogation sur le corps de l'homme, sur sa corporéité» (p. 198). Ces ruptures font sortir de la longue tradition, mise en scène par Three Men and $a$ Baby, de la dénégation du corps masculin, «réduit à être une machine musculaire» (Peter fait de la musculation, du jogging) et «une machine à éjaculer» (p. 198) (Jack est une giant gland).

Dans le film de Coline Serreau, c'est cette crise de la masculinité qui est montrée. Dans celui de Leonard Nimoy, c'est le mouvement de l'adolescence à une pseudo-maturité qui est articulée, celle de la responsabilisation: mouvement qui sera simplement accéléré par un conflit provoqué par le corps étranger du bébé dans l'organisme juvénile du boy américain. Il y a donc eu distorsion et récupération dans la relecture de Nimoy: la crise devient simplement conflit. La masculinité traditionnelle reste glorieuse et acquiert, en fait, un surplus de gloire par la responsabilité prise. Le transfert transatlantique opéré passe par une masculinisation, car Nimoy est non seulement américain mais aussi mâle. Il y a donc eu dans le remake un double processus de transposition. Nous démontrerons cette idéologie du statu quo en jeu dans Three Men and a Baby, en examinant le rapport des sujets masculins aux femmes dans les deux films: en effet leur rôle de potentielles mères de l'enfant abandonné est le punctum par rapport auquel le sujet masculin paternant devra se replacer.

Se séparer de la Femme pour advenir comme «nouveau père»:Trois hommes et un couffin.

L'enfant, dit Christiane Olivier, rappelle à l'homme un autre luimême, "petit garçon qui a vécu auprès de sa mère» (p. 203). Ainsi, pour savoir quoi donner à l'enfant, faut-il d'abord se rap- 
porter à soi-bébé: "We were babies once, for God's sake, what did we eat?" demande Michael à Peter; de même, quand la mère de Jack porte l'enfant, celui-ci n'est plus père putatif, mais redevient petit garçon:

MOTHER. — I had her laughing for a minute!

JACK. — Just like me, eh?

Mais ce petit garçon-là a aussi «tout fait pour ne pas s'assimiler à elle» (p. 203). La peur de l'homme est donc de se montrer femme: de là vient son refus «d'assumer la paternité réelle, c'est-à-dire le corps à corps avec l'enfant» (p. 203). Ainsi, Jacques, au téléphone avec Sylvia, la mère qui a abandonné le père à sa «condition», affirme:

Qu'est-ce qui me prouve que je suis son père? (...) les dates concordent, les dates concordent, ça prouve rien. Alors comme ça, toi, tu le sais. Eh bien moi, je le sais pas. Alors, tu reviens, tu reprends le gosse (...) (clack! il se fait raccrocher le téléphone au nez).

Se réveillent alors les «vieilles défenses organisées lors des premières années face à sa propre mère maternante» (p. 203). Â la découverte du couffin derrière la porte, c'est la panique:

MiCHEL. - Téléphone à la mère de Jacques.

PIERRE. - Ah! non! Une emmerdeuse pareille en plus. Je vois déjà le bombardement de questions. Tu te rends compte si elle arrive avec sa valise, Jacques nous le pardonnerait jamais.

MiCHEL. — Je peux peut-être l'appeler, la mienne de mère?

PIERRE. - Ah! s'il te plaît! tu laisses les mères là où elles sont. Si on laisse les mères s'engouffrer dans ceci, on en a pour dix ans. D'abord, c'est simple, si une femme arrive ici, je déménage... ça a toujours été convenu comme ça.

D'abord, le contrat entre eux est un contrat misogyne: pas de femme plus d'une nuit dans l'appartement. Ensuite, Coline Serreau fait de la mère de Jacques une femme du «nouveau» troisième âge, qui a des copines et qui voyage. Son impossibilité de prendre soin du bébé donne lieu, d'ailleurs, à une des scènes les plus comiques du film, montrant la déconfiture de Jacques devant la joie de vivre de sa mère: "Qu'est-ce qu'elle a comme amies! Putain de carteVermeil ${ }^{10} \ldots$ à son âge, faire la bamboula aux Caraïbes!» Elle, elle s'est détachée de lui et de la fonction maternante. 
Dans le film de Serreau, cette séparation «convenue» d'avec le monde des femmes (amantes, mères et plus tard la nurse qui se fera évacuée en bonne et due forme) est la trace d'une lutte identitaire: en effet, il s'agit pour le sujet masculin français de ne «jamais être assimilable ou assimilé aux femmes (...). [Cette lutte] est la trace de son anti-identification ou misogynie» (p. 204). La mère omniprésente, le père souvent absent font en sorte que le sujet masculin s'est «construit davantage à l'envers de la mère qu'en rapport avec le père ${ }^{11}$ » (p. 204). Le scénario français crée trois hommes qui se sont débarrassés symboliquement de toute femme: ils sont prêts à aller au-delà de leur misogynie effective et déclarée pour advenir à une nouvelle masculinité (non phallique).

L'enfant sera ainsi radicalement séparé de toute présence féminine qui pourrait s'approprier son corps: car, dans nos sociétés, c'est cette présence physique continuelle de la mère qui a eu, pour conséquence immédiate, la «fixation [inéluctable] à la mère (...) doublée d'une conséquence plus lointaine: il faudra se défaire de cette mère, il faudra engager la guerre contre la femme» (p. 205). Ainsi, dans Trois hommes et un couffin, pour advenir comme sujet masculin et père paternant, l'ennemi, c'est d'abord la Femme. Mais il n'y a pas confusion entre La Femme (figure fantasmatique puissante) et les femmes (corps vivants/désirants et sujets en devenir) dans le film français, à l'inverse du film de Nimoy. Les colères, les insultes misogynes fusent dans le film français. Jamais on ne se sent, spectatrice, mise en jeu. Le sujet masculin est en train de faire face à La Mère toute puissante en s'appropriant ses gestes: c'est avec le patriarcat matrilinéaire qu'il se bat.

Une fois la Femme évacuée, le père jusque-là «empêché» par elle, va pouvoir y trouver son compte de plaisir et de tendresse. Il n'est plus celui qui sépare l'enfant de la mère, tiers symbolique, simple nom-du-père, etc., comme dans la psychanalyse «patriarchique» de Freud et de Lacan: son «énorme» absence, au lieu d'être dénoncée par cette psychanalyse, nous dit Olivier, a toujours été considérée comme ayant une fonction structurante primordiale pour le sujet (p. 204). Par sa multiple présence physique dans le film (trois pères tout à coup là où il n'y en avait aucun), le signifié père jusque là vide (puisqu'il n'y avait que du signifiant ${ }^{12}$ ), est soudain rempli de présence, soudain réellement «perceptible par les sens de l'enfant» (p. 204).

Ainsi, le projet de Coline Serreau s'insère dans cette problématique: montrer que même si «apparemment [les hommes] font pour l'enfant les mêmes choses que la mère, leurs actes restent masculins: un père qui pouponne son enfant fait du masculin et non du féminin» (p. 204). D'où la pluralité des sujets montrés. Le film a en quelque sorte valeur de démonstration: trois est alors 
l'échantillon minimum pour conclure au bien-fondé des résultats, pour faire admettre l'axiome d'un paternage qui ne soit pas féminisant.

\section{Faire comme dit Maman et devenir «responsable»: Three Men and a Baby}

\section{La double contrainte}

Dans la version américaine masculine, le fils ne se détache pas de la Femme, de la Mère, ni dans son imaginaire, ni dans le récit. Jack fait venir sa mère et lui demande de garder l'enfant pour lui, car il ne sait pas comment faire. Mais la mère, femme du monde, lui répond en tenant le bébé:

LA MÈRE. - Some people live all their lives without having anything so wonderful to show for it... I'm going to do the most wonderful thing for you... Absolutely nothing.

JACK. - Muuum...

LA MÈRE. - Jack, you've always run away from responsability. Now, you have to turn and face it.

JACK. — Mum, I'm a screw-up.

LA MÈRE. - You were a screw-up. Now you are a father ${ }^{13}$, and you'll be a fine one.

JACK. — You think so?

De même, la copine steady de Peter (remarquons qu'il n'y a pas de steady dans le film français) refuse de s'en occuper et rejoue la position de la mère vis-à-vis de lui:"You're a very big boy and very capable. You're going to go through this just fine!" Elle trouve aussi très comique d'entendre son gros bonhomme de Tom Selleck chanter une berceuse au bébé14. Les femmes (figures multiples de la même Femme-Mère) ont ainsi un regard infantilisant, amusé et ironique. Elles obligent le fils-garçon à devenir père et elles observent et commentent. Ainsi restent-elles détentrices d'un savoir secret, du pouvoir phallique: non castrées dans l'imaginaire, elles restent objet de désir du petit Edipe. Jack gardera l'enfant pour faire plaisir à sa mère (la vision œedipienne masculine jouée dans le fantasme du film créant par là le désir de la mère: la mère du film, rappelons-le, étant un personnage dans l'imaginaire du sujet œdipien masculin). 
Puisqu'elle est, somme toute, une réponse à l'injonction maternelle ("Be a father!"), Jack devient père par double contrainte: il n'a ni choix ni possibilité de sortie. La pseudo nouvelle paternité reste forcée, artificielle, abstraite. Jack l'exprime ainsi: "I'm an actor, I can do a father!" Et Peter: "I'm an architect. I build fiftystorey sky-scrapers. I design cities of the future, I should be able to put this goddam diaper."

On entre ainsi dans le domaine du how to et du should do de la moralisation. Tout ce qu'ils gagnent de l'expérience est un surplus de devoirs et par là un supplément de statut viril. Les gadgets et les arrangements topographiques vont remplacer tout processus de maturation, c'est-à-dire de sortie de la phase œdipienne: on trimbale l'enfant au chantier, à la répétition théâtrale ${ }^{15}$, etc. Ainsi, la figure maternelle reste toute-puissante: "[Mary] needs a full-time mother", dit-on avant de proposer à cette mère d'emménager carrément avec les trois hommes: ce qui profite autant à ces derniers et ne remet pas en cause les fonctions sociales et imaginaires de mère et de père. Ce qu'ils gagnent de l'expérience, c'est une nouvelle liste de should et should not, dans la culpabilisation: cette dernière joue le lien masculin au père comme étant position du rival œdipien, position de la loi qui est infiniment rejouée dans le rapport à l'autre homme, dans le regard phallique comme castration (imaginaire). Les trois héros continuent leur route vers leur telos matérialiste, fétichiste (l'objet à la place de tout autre vivant, «différent»). La dernière image du film montre le dessin ajouté de la mère et de l'enfant sur leur mur: leur image à eux n'a pas changé, ils sont encore prêts pour toutes leurs conquêtes donjuanesques juvéniles (c'est-à-dire accumulation de femmes-objets fétiches).

Les hommes restent ainsi gender-defensive et la lutte des sexes continue. La résistance à la féminisation - ce qui rend comique le film français -, les défenses masculines, ont été non reconnues et donc non résolues dans la version américaine. Le faire sublimatoire (l'action, l'accumulation, la répétition des gestes ${ }^{16}$ ) permet l'effacement du désir (des trois sujets masculins) vis-à-vis du corps paternel, pulsion érotique refoulée par un déplacement: c'est le désir de l'enfant, l'enfant comme sujet, qui est non représenté (le sujet masculin s'identifie à l'enfant, et s'interdit, à travers lui, un rapport possiblement érotique vis-à-vis du corps paternel: il oscille donc entre deux positions imaginaires enfant/père). La paternité se pose comme question de maîtrise vis-à-vis de l'objet-enfant, l'argent et l'inventivité sublimant toute angoisse possible vis-à-vis de l'enfant-sujet: "I'll give you ten dollars if you stop crying", dit Michael au bébé; "I'll give you a thousand dollars if you'll [change her]!" dit Peter à Michael. C'est-à-dire je remplacerai ton corps 
pleurant, urinant, par un ersatz, un fétiche sec et rond, complet sans la béance que la demande instaure, sans ton altérité.

\section{L'action obligatoire}

Actif compulsif, le mâle de Nimoy doit se débattre contre la passivité (n'en finit pas de se séparer de la Mère). Ainsi, le chagrin causé par le départ de la petite va durer dans la diégèse, un aprèsmidi et un gros cinq minutes dans le temps filmique. Car le centre du film s'est déplacé en traversant l'Atlantique. La poursuite des trafiquants de drogue (corps d'hommes en action) est devenue le centre du film ${ }^{17}$, tandis que les trois Français, eux, se complairont dans la mélancolie, la grande déprime, les maladies psychosomatiques pendant un bon bout de temps, sans jamais agir ni en parler (ce sont alors les corps qui parlent): le centre narratif du film étant le départ de l'enfant, c'est-à-dire le départ réel de l'autre. Le corps à corps en est interrompu, les signes du manque indiquent que le lien a été tranché douloureusement. Jacques montrera la profondeur de la «crise ontologique» quand il demandera à Michel: «Je voudrais savoir pourquoi je vis. Tu sais, toi, pourquoi tu vis?» L'inconscient masculin (le désir) français aura eu les moyens de se faire savoir, dans les silences prolongés de certaines scènes, dans l'incohérence des explications, dans les colères, les «engueulades maison», les postures des corps, dans l'obscurité de l'appartement ${ }^{18}$. La fin des résistances est ainsi montrée par la parole débridée, la perte de maîtrise: une parole autre se met en place. Mais surtout, le sujet masculin de Nimoy est bien loin dans le questionnement de la sexuation culturelle de celui de Serreau: ainsi, cette tirade de Jacques, le père biologique, a été supprimée dans le remake ${ }^{19}$ :

JACQUES, saoul, une bouteille à la main, «enceinte» d'un oreiller, parlant au policier en chômage sur le banc du parc. - Si j'étais Dieu, je fabriquerais Adam avec la côte d'Êve, pas l'inverse. Comme ça les choses auraient été claires dès le début. On nous aurait pas fait croire que quelqu'un sort de notre côte, parce que rien sort de notre côte à nous, jamais. Tout juste de notre queue, et encore y a tout à faire après. Nous, ce qu'on sait fabriquer, c'est des buildings, des avions, des voitures, c'est utile, remarquez... C'est même pas qu'on a voulu nous le faire croire, si ça se trouve, c'est nous qu'avons voulu le croire. Non, il faut se rendre à l'évidence, personne ne sort de notre côte.

Dans le film de Nimoy, tout est littéralement en pleine lumière, pour mieux y cacher la résistance. L'inconscient américain a un surmoi à la taille du pays et de sa masculinité «stéroïdée». L'homme maternant ne peut que cabotiner, car il se sent observé: "I keep waiting for Candid Camera to walk through the door!" Même quand il commence à y prendre plaisir et qu'il est seul de- 
vant la caméra, il n'en finit pas de jouer pour l'homme-absent: le quatrième homme (celui qui punit). Chez Serreau, on se laisse aller à des gazouillis subitement stoppés, évidemment, quand le colocataire entre. L'homme américain ne se laisse pas aller, ni à détester franchement les femmes, à évacuer la figure maternelle phallique, ni à ridiculiser le père en son absence ${ }^{20}$, ni à toucher l'enfant sinon pour lui faire quelque chose. Il est trop bien-pensant, c'est-à-dire dans l'autocensure.

"We should be her family", déclare Peter-Tom Seleck à la fin. Un autre should pour tout arranger, résoudre le conflit: rien n'a changé, le devoir, l'éternité de l'être (be et pas become) et le concept sacro-saint de famille américaine, légèrement éclaté, mais si peu. Trois garçonnets jouant au papa sous le regard de maman, au lieu d'un père absent, c'est alors simplement une autre forme de l'absence. Les hommes américains suivent à la lettre les conseils de leur maman indétrônable.

Est-ce parce que la mère phallique américaine est difficilement «tuable»? Est-elle moins opprimée que la mère française par la «patriarchie», donc moins totalitaire dans son pouvoir maternel? Ou plutôt, les hommes américains n'ont-ils pas été rendus coupables par le féminisme humaniste des Américaines, un féminisme qui continue la tradition puritaine de la censure de l'imaginaire, car il ne croit pas à l'inconscient? Ainsi, le quatrième homme du film américain serait une espèce de surmoi maternel/féministe (détenant encore le phallus), un patriarche, travesti en femme pour continuer à censurer, c'est-à-dire faire le père?

\section{Puritanisme et perversion}

Et la conséquence première de la censure, c'est le plaisir du péché qui a pour corollaire la perversion. Elle s'immisce, l'obsession du sexe, comme dans tout ce qui vient d'Hollywood. L'objet de la répulsion et de désir, dans le film, ne l'oublions pas, est de sexe féminin, elle s'appelle Mary/Marie.

Pourquoi, d'ailleurs, avoir choisi une petite fille? L'enfant français n'est-il pas simplement un bébé-fille parce qu'ainsi, elle «est très loin de l'homme» et «très loin de la femme envers laquelle celui-ci vit le... désir» (Chancel, p. 209-210)? Elle est potentiellement féminine, objet d'amour avec laquelle toute la relation à l'autre sexe va être repensée: ainsi à la fin, la Sylvia française s'endort dans le berceau de sa fille, en suçant son pouce, rejoignant ainsi l'enfant et la femme dans une même posture, dans un continuum. C'est une reconnaissance de confiance qui fait des trois hommes des vrais pères ayant réussi à trouver leur paternité sans avoir copié, sans avoir fait la mère: et tout à coup ça glisse sur l'axe de la sexuation, il n'y a plus de place fixe. 
Dans Three Men and a Baby par contre, la relation d'objet avec la petite fille est marquée par la défense et la séduction. La manière de se sortir de l'angoisse, causée par la représentation (refoulée) du désir du père (dans les deux sens), c'est alors de constituer l'enfant comme une extension de l'ego masculin (pour ne pas dire de son phallus). Les exemples sont nombreux. Ainsi Michael, après avoir montré sa chambre au bébé (comme s'il s'agissait d'une possible maîtresse), s'allonge sur son lit, le bébé sur les genoux; il ouvre la télé, comme par hasard: c'est Doctor Ruth qui parle d'orgasme et de pénis. "No, no, no! s'écrie-t-il en éteignant le poste, You don't want to hear that!21" L'enfant est à la fois objet sexuel phallique (position sur le corps du jeune homme) et dénié comme tel dans un va-et-vient entre le oui et le non. Â un autre moment, dans le parc, celui qui porte le bébé, retirant un surplus de virilité de cet objet montré et porté, est aussitôt assiégé par des dizaines de filles; scène muette jouée sur des paroles de chanson ambiguës: "Little Baby let me hold you tight!" Enfin, les paroles suivantes sont fort révélatrices:

MICHAEL, avant de changer la couche du bébé. - Peter, this is a girl, should we be doing this?

PETER, au policier qui veut voir l'enfant. - She might not be decent.

\section{LE POLICIER. - I don't think she'll mind!}

Ces boutades sont évidemment là pour faire rire le public, au premier degré, c'est-à-dire par la sexualisation (c'est-à-dire être l'objet de désir masculin, seulement) et la sexuation figée, évidente, automatique 22 d'un bébé supposé "pur et innocent». Si dans le film de Serreau, le sexe de l'enfant n'est jamais mentionné, il doit l'être pour rassurer l'homme version Hollywood, car si les mâles doivent rester des sujets pleins, monolithiques, l'enfant doit être posée comme sujet pleinement fille, pleinement sexuée dès la naissance, biologiquement donc. Asexuer l'enfant, ne pas mentionner son sexe, c'est-à-dire sa nudité, serait pour le mâle hollywoodien courir de grands risques: le premier d'être accusé de pédophilie (mais il l'est, pédophile, puisqu'il s'en défend si mal, puisqu'il ne peut pas ne pas voir le sexe); le deuxième risque (et il est peut-être pire) d'être asexué, c'est-à-dire femme, car il n'y a que deux pôles dans la dichotomie inébranlable de cette idéologie du statu quo. L'action, la violence (attaque du colocataire qui rentre la nuit, mise en abyme du film policier du milieu, etc.) subliment le risque d'asexuation, donc de féminisation. La mise en scène obsessive de 
la dénégation du désir sexuel, forcée par la morale puritaine, inscrira donc en filigrane la perversion du père comme ce qui coule de l'humanisme américain. Puisque le sexe est partout, se dit-on, il faut alors le saisir et l'incarner par le corps, pour reprendre les propos de Jules Chancel, «d'une espèce d'eunuque aux biceps [durs et] saillants» (p. 210): le bébé devenant ce phallus séparable du corps et par là désirable et pourvoyeur d'une possible jouissance auto-érotique montrable dès lors qu'elle est déniée.

La sexuation (souvent stéréotypée) du héros américain est la condition de son asexualisation: pas de glissement vers une possible bisexualité, une androgynie qui donnerait à jouir, et donc pas de reconnaissance du pouvoir de la féminité, du corps et de la sexualité des femmes. Ainsi le Jacques français pousse cette belle tirade sur l'impossibilité pour les hommes d'avoir de l'autre en eux; le Jack américain, lui, se contentera de s'habiller en femme enceinte seulement pour semer la police, cachant sous son manteau le vrai bébé. Et le tour est joué! On ne pose pas de questions sur son rapport à l'altérité (enfant et/ou femme): l'illusion de la production d'autre passera par l'action sur l'autre: les bad guys livrés à la police; et le mâle reste seul face à son miroir.

Ainsi, Tom Selleck se déguise en hétérosexuel viril pour jouer au papa avec un corps déjà tout façonnné par Magnum $P$. I. L'effet d'adresse est ici identifiable comme cette «hollywoodisation» qui refond l'étrangeté possible du film original pour être à la fois rentable et lisible: rentable parce que lisible pour le «grand public». Le choix des acteurs, marqués déjà comme «figures types» de la masculinité américaine et de la comédie légère amuse: le rire viendra de la transposition de héros de télévision dans un cinéma où le travestissement ne fait que les révéler dans leur autosimilitude. Le même se reproduit dans une halloween où on pourrait jouer au couple homosexuel avec, pour excuse, un bébé tombé du ciel; où on pourrait faire la Femme (la Mère) en somme, en s'ajoutant, sur le corps, des traits de la mascarade du féminin patriarcal, comme si c'était là la question des sexes, un problème de robinet, de soustraction et d'addition!

Ce sujet masculin-là et la multiplication par trois de son narcissisme colossal a un corps «barré» par la dénégation de son vide et donc de sa possible jouissance. L'homme américain de Nimoy non seulement ne sait pas qu'on ne la lui coupera pas s'il désobéit à sa mère, mais surtout il feint d'ignorer que rien jamais ne sortira de son ventre. On n'y sait pas non plus qu'un père, c'est autre chose qu'un homme jouant à la Mère: dans Three men and a Baby, l'homme viril s'est simplement déguisé en maman, sous la double contrainte maternelle (œdipienne phallique). On reste le fils de sa mère, sans remettre en cause, en pouponnant, sa place imaginaire à 
elle. Et donc la question de la sexuation ne se pose pas, comme dans le film de Coline Serreau, à la façon d'un construit de positions imaginaires familiales et des postures corporelles culturelles et historiques. Nimoy aura fait une «re-vision» du regard attendri que Serreau portait sur les nouveaux hommes nés des mouvements et des analyses féministes: il aura transformé leurs corps en simples supports pour rejouer l'idéologie patriarcale américaine. La crise bénéfique du sujet masculin n'a pas passé la rampe: cette récupération de Nimoy et de sa machine est décelable comme la tension de cette américanité qui continue de se rêver avec, pour porte-drapeau, ce jeune homme américain type, naïf adolescent musclé qui tient le fort assiégé de la masculinité, une masculinité déniée comme foncièrement homosexuelle. Ce qui a été posé dans Trois hommes et un couffin et qui n'a pas eu lieu dans le remake, c'est la possibilité future d'une intersubjectivité dans la différence sexuelle, d'une véritable hétérosexualité.

\section{University of Western Ontario}

\section{NOTES}

1 Des bruits courent selon lesquels, après qu'elle eut réalisé qu'on avait choisi Tom Selleck pour jouer le plus paternel des pères, Serreau aurait claqué la porte: le corps de Selleck étant certainement trop «chargé» de sens, déjà mythique pour le public américain, ce choix transformait irrémédiablement le projet «politique» initial de Serreau.

2 Nous devons ce bref rappel de la position freudienne à l'article de Paul-Laurent Assoun, «Fonctions freudiennes du père», Le Père: Métaphore paternelle et fonctions du père: L'Interdit, la Filiation, la Transmission (collectif) (Paris: Denoël, 1989) p. 25-51.

3 Voir le travail que fait Christine Delphy (chercheure au CNRS) à ce propos; entre autres sa communication à l'ACFAS (UQAM, 16-05-1989). Ce travail se situe au niveau d'une analyse marxiste très efficace des présupposés des anthropologues féministes et de leurs «fantasmes» (bien que le mot ne soit pas utilisé par Delphy, car elle ne travaille pas au niveau de l'imaginaire, ni des rapports inconscients).

4 Terme d'ailleurs extraordinairement ironique, car que reconnaît-il? Sa participation historique à la conception, l'enfant en étant alors le résultat? Reconnaît-il alors la fidélité de sa femme, donc sa puissance génératrice à lui? Ou reconnaît-il la bonne foi de sa femme qui lui présente l'enfant comme le sien?

5 Voir à ce sujet Jacques Lacan, «Le stade du miroir comme formateur de la fonction du je: telle qu'elle nous est révélée dans l'expérience psychanalytique», Écrits (Seuil, 1966) p. 93-100.

6 Nous renvoyons, ici, au travail en cours de Martin Lefebvre, de l'University of Alberta, qui reprend cette question à partir d'une relecture attentive de la sémiologie peircienne et de son application au cinéma. 
7 Incidemment, le Français emmènera avec lui une bouteille de whisky, l'Américain une bouteille de Bordeaux 82, "which works every time"; l'exotisme de l'Outre-Atlantique fonctionne dans les deux sens.

8 Il s'agit de femmes sociales (fonctionnelles). C'est ce qui est en jeu dans l'original français: montrer que la mère est un rôle culturel et non biologique.

9 «De l'identique à l'identité: entretien avec Geneviève Delaisi de Parseval», Autrement. 61 (juin 1984) p. 198. Voir aussi son livre La Part du père (Paris: Seuil, 1981).

10 Carte de réduction sur les chemins de fer pour personnes du troisième âge.

11 Notons que le père est carrément absent pour les fils de Trois hommes et un couffin et mentionné en passant comme semblable au fils (et déjà mort) dans Three Men and a Baby. La mère de Jack à son fils: "Your father, God bless his soul, he was a screw-up and he turned out just fine! Don't you remember?"

$12 \mathrm{Il}$ faudrait problématiser et contextualiser, dans la relecture lacanienne, cette binarité saussurienne. Je ne suis pas sûre qu'Olivier les emploie à bon escient, car comment le contact sensuel avec le père peut-il être du signifié? Celui-ci, chez Lacan comme chez Saussure, est langage, mais chez le premier il est position et n'est pas contemporain du signifiant, il est produit par lui. Il signifie l'assujettissement du sujet à la loi du signifiant, c'est-à-dire au désir comme castré. Ainsi, là où il y a du corps, il n'y a pas de signe, pour citer Gilles Thérien (Séminaire de sémiologie, UQAM, 1989), puisque le corps (ici du père) absenterait son signe, signe qui est toujours à la place d'une absence: d'où la difficulté de penser «le corps du père» ou même le corps tout court. D'ailleurs, pour la psychanalyse, il serait (comme objet du désir) ce qui pourrait être là ou le signifiant (nom-du-père) a pris sa place. La structure (du nom-du-père) est toute-puissante et la possibilité même de penser le corps paternel demande de pousser un peu plus loin les possibilités subversives de la construction lacanienne: ceci est très faisable, puisque «Homme» et «Femme» sont des positions sur la chaîne des signifiants et non des rôles biologiques, d'où la déclaration qu' «il n'y a pas de rapport sexuel» (voir Jacques Lacan, «La fonction de l'écrit», Encore (Paris: Seuil, 1975) p. 29-38, particulièrement p. 36.

13 Ici, le fils est nommé père par la mère: I'Edipe masculin prend un angle nouveau, celui d'être le père de l'enfant de la mère, par la parole de la mère.

14 Et là elle devient l'incarnation de toutes les admiratrices de Magnum qui rient (tendrement) avec elle.

15 L'enfant y apparaît premièrement comme un «petit même» (donc masculin): Peter habille Marie avec un petit casque identique au sien; pendant la période de «mélancolie», à la fin, ce petit casque sera retrouvé sous le grand casque de papa: l'enfant est intérieur au père (maternité imaginaire), un petit Peter tout fait. Deuxièmement, attaché dans le dos de Jack l'acteur répétant, l'enfant est «excroissance» du corps, il en fait partie intégrante. Ce cabotinage (comique pour ces mêmes raisons: la paternité maladroite a quelque chose de touchant) revient à «éliminer» l'enfant, à faire de lui (d'elle en l'occurrence) un semblable indifférencié (image de soi, partie de soi). Nous avons là affaire à des stratégies de refoulement de l'altérité: cette dernière devrait passer comme la représentation de la demande de l'enfant, enfant devenant sujet par cette demande (à ne pas confondre avec besoin: on ne parle que de celui-ci dans le film américain - besoin de nourriture, de soins corporels). La demande, elle, parle de désir, elle est rapport intersubjectif. Mais la satisfaction de cette demande devrait mettre en place, en face, le désir du père pour (le corps de) l'enfant. Représenter l'altérité, sexuée donc, fait risque (nous y reviendrons). 
16 Voir le déplacement répété du biberon en accéléré, comme un mime du lancement de la balle au base-ball, ratages, écrasements du biberon par terre subséquents: ces mouvements inscrivent un rapport sadique-anal à l'objet, rapport d'ailleurs sensible dans la destruction presque obligée de voitures, maisons (propriétés privées valorisées aux États-Unis) dans le film commercial hollywoodien.

17 Le surmoi paternel terrible règne et la moralisation continue: dans le film de Serreau, on rend les sacs de drogue aux négociants; les péripéties tournant autour de la couche du bébé à jeter dans une poubelle d'un jardin public; dans le film de Nimoy, la machine hollywoodienne en fait «un film dans le film» à la tonalité Magnum $P$. I., où c'est Tom Selleck qui initie l'action: on l'a rendu juste un peu plus timide! Le paradigme est le même. La police représente la loi surmoïque. Il est intéressant de noter que, chez Serreau, on ne dénonce pas le «frère» — trafiquant, qui reste sympathique mais que la figure «paternelle» du flic est ridiculisée par un Michel qui joue à l'homosexuel, donc dans la position de la fille séductrice vis-à-vis du père: position subversive de l'autorité, il refuse de faire le «fils", de risquer la «castration». La figure paternelle «traditionnelle» s'en trouve déchue. C'est Michel (le plus fille des trois) qui trompera le flic. Rappelons aussi que ce flic-là reviendra plus tard, pour «savoir» comment il a été trompé. Sa figure à ce moment n'est plus celle de l'autorité: il est en chômage, renvoyé par le «père». Il entre alors dans le camp des "nouveaux hommes», ceux qui ont dépassé l'angoisse de la castration.

18 Devenu scène obscure du désir.

19 Ce qu'il en reste, c'est Ted Danson, avec un oreiller sous le chandail devant son miroir, pendant un bref clin d'œil qui a quelque chose qui ne passe pas la barre du cabotinage.

20 Car ridiculiser, c'est imiter, rire du corps, des postures: le père n'a pas de corps pour Nimoy et ses acteurs; le corps de l'autre homme est un corps-objet sadique-anal, non encore séparé de soi: blessé, battu, agressé, poursuivi, détruit.

21 En d'autres termes: "I don't want you to want..."

22 Le bébé américain est habillé de rose, dans un couffin rose: pas de doute possible.

\section{OUVRAGES CITÉS}

Assoun, Paul-Laurent. «Fonctions freudiennes du père». Le Père: métaphore paternelle et fonctions du père: L'Interdit, la Filiation, la Transmission (collectif). Paris: Denoël, 1989, 25-51.

Chancel, Jules. «Le corps de B». Autrement. 61 (juin 84) p. 209-210.

Delaisi de Parseval, Geneviève. «De l'identique à l'identité» (entretiens). Autrement. 61 (juin 1984) p. 198.

Olivier, Christiane. «Pères empêchés». Autrement. 61 (1984) p. 201-212. 